\section{MS13-P13 Crystal structure of a DNA catalyst}

Almudena Ponce-Salvatierra ${ }^{1,2}$, Claudia Höbartner ${ }^{2,3}$, Vladimir Pena $^{1}$

1. Macromolecular Crystallography research group, Max Planck Institute for Biophysical Chemistry, Göttingen, Germany

2. Max Planck research group Nucleic Acid Chemistry, Max Planck Institute for Biophysical Chemistry, Göttingen, Germany

3. Institut für Organische und Biomolekulare Chemie Georg-August-Universität Göttingen, Göttingen, Germany

email: aponces@gwdg.de

In structural terms, the differences between functional RNA and DNA molecules are rather dramatic. RNA adopts complicated folds within various ribozymes and riboswitches. This complexity relates directly to the variety of functions that RNA has. For example ribozymes, like group II introns or the ribosomal RNA, are organized by means of tertiary interactions to an overwhelming level of complexity, only comparable to the one of proteins. Despite the fact that DNA and RNA are very similar in terms of building blocks, DNA has been described in much simpler conformations that lack long-range tertiary interactions. One reason for the conformational simplicity of DNA appeared to be the absence of the 2'-OH group, resulting in greater chemical stability of DNA and rendering it suitable for its role as a long-term storage molecule. It is indeed the presence or the absence of the 2'-OH group what causes the main differences in terms of backbone conformation between DNA and RNA. When the 2'-OH is missing, the sugar conformation of the ribose is $\mathrm{C}^{2}$-endo, leading to the B-type of helix preferentially. On the other hand, the presence of the 2'-OH leads towards the C3'-endo conformation and thus to the A type of helix.

In recent years, synthetic DNA catalysts came under the spotlight as very intriguing constructs, since enzymatic activity is expected to depend on the ability to fold into compact tertiary structures, as in the case of ribozymes or protein enzymes. Therefore, to understand how DNA molecules are organized in $3 D$ to achieve enzymatic properties, we decided to investigate a DNA catalyst able to ligate two RNA molecules. Here we report the structure of this deoxyribozyme in complex with the RNA product.

The structure reveals an autonomously folding unit stabilized by a wealth of tertiary interactions, including extensive base stacking and alternative base-pairing. It exhibits various features that were never observed in DNA molecules. The structure unifies a large body of biochemical data and it clearly demonstrates that DNA structures can be much more intricate and versatile than generally appreciated. The structure unveils a novel organization of the catalytic center and provides the first insight into the manner how a DNA molecule catalyzes RNA ligation.

Finally, on the basis of the structure, we have engineered the original RNA-ligase deoxyribozyme to make a general RNA-ligase.

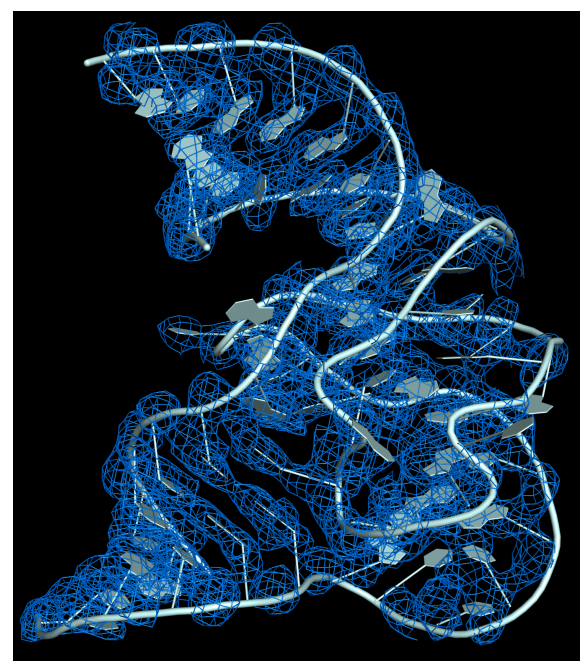

Figure 1. Solvent flattened electron density map contoured at 1 sigma level with light blue trace of the backbone and nucleobases.

Keywords: deoxyribozyme, DNA catalysis, RNA-ligase, rational design, alternative base-pairing, long-range interactions, structure 